\title{
Basics of geomatics
}

\author{
Mario A. Gomarasca
}

Published online: 27 July 2010

(C) The Author(s) 2010. This article is published with open access at Springerlink.com

\begin{abstract}
This paper presents the volume "Basics of Geomatics" (Springer, ISBN 978-1-4020-9013-4). The volume introduces in a systematic way the complex topics and techniques that can be assembled under Geospatial Information, namely, geodesy, cartography, photogrammetry, remote sensing, informatics, acquisition systems, global positioning systems, digital image processing, geographic information systems, decision support systems, and WebGIS. It describes in detail and at an accessible level the state of current knowledge. As such, it will serve as a working tool not only to geoscientists and geographers but also to engineers, architects, computer scientists, urban planners, specialists in geographical information system, remote sensing, forestry, agricultural science, soil science geometry, environmental scientists, and managers. Applications can be found in security, risk management, monitoring, infomobility, geopositioning, food traceability, etc.
\end{abstract}

\section{Introduction}

This paper presents the volume "Basics of Geomatics" (Springer, ISBN 978-1-4020-9013-4).

From classical geography, scientific activities in Earth Observation have undergone a rapid expansion, and more and more economic sectors tend to employ territorial data acquired by ground survey, global satellite positioning systems, traditional and digital photogrammetry, and mul-

\footnotetext{
M. A. Gomarasca $(\bowtie)$

National Research Council of Italy,

Institute for the Electromagnetic Sensing of the Environment,

Via Bassini 15,

20133 Milan, Italy

e-mail: gomarasca.m@irea.cnr.it
}

tispectral and hyperspectral remote sensing from airplane and satellite, with images both passive optical and active microwave (radar) at different geometric, spectral, radiometric, and temporal resolutions, although there is still only limited awareness of how to use all the available potential correctly. The resulting data and information are represented in digital and numerical layers managed in Geographical Information Systems and Decision Support Systems, often based on the development of Expert Systems.

Such a large amount of data must necessarily be organized, processed, handled, and used without delay for a correct representation of the territorial situation.

These elements must be processed in an interdisciplinary and interoperable manner, and the discipline of geomatics (geos: Earth, matics: informatics) can satisfy such requirements.

The term geomatics was created at Laval University in Canada in the early 1980s, based on the concept that the increasing potential of electronic computing was revolutionizing surveys and representation sciences and that the use of computerized design (video diagram) was compatible with the treatment of huge amounts of data. That period's revolutionary intuition was based on the geographical location of each object on our planet.

Geomatics is defined as a systemic, multidisciplinary, integrated approach to selecting the instruments and the appropriate techniques for collecting, storing, integrating, modeling, analyzing, retrieving at will, transforming, displaying, and distributing spatially georeferenced data from different sources with well-defined accuracy characteristics and continuity in a digital format.

Erected on the scientific framework of geodesy, it uses terrestrial, marine, airborne, and satellite-based sensors to acquire spatial and other data. 
Some initiatives are presently developing worldwide using geomatics disciplines and techniques for the regulation of GeoSpatial Information or more simply GeoInformation and for the adequate use of Earth Observation data for studying and managing environmental hazards and risks.

Several countries, following common fundamental guidelines and procedures, are developing a Spatial Data Infrastructure. At planet level, the dream is to realize a Global Spatial Data Infrastructure (GSDI) capable of managing heterogeneous sets of data and to overcome the chronic absence of interoperability among databases. This goal can be reached by implementing several SDIs at the local, national, and continental level and harmonizing them in a global context.

One practical example is represented by the Infrastructure for Spatial Information in the European Community, a European Union Directive that entered into force on May $15,2007$.

Other initiatives promote the collective effort for a better Earth environment, by increasing our understanding of the Earth's dynamic processes and enhancing forecasts of our environmental conditions. The Group on Earth Observations was formed to undertake this global effort, and the Global Earth Observation System of Systems (GEOSS) was established on February 16, 2005, with the scope of addressing all nations involved to produce and to manage their information in a way that benefits the environment as well as humanity by taking the pulse of the planet.

As an example, the European initiative Global Monitoring for Environment and Security is intended to propose solutions for an articulate, centrally coordinated system for risk management at a European level, contributing to GEOSS.

The disciplines and techniques constituting geomatics are:

- Computer science: to represent and process applicable information through the development of technological instruments (i.e., hardware) and of methods, models, and systems (i.e., software)

- Geodesy: to determine the shape and size of the Earth; it defines on the one hand the surface of reference in its complete form, the geoid, as well as in its simplified form, the ellipsoid, and on the other hand the external gravitational field as a function of time

- Topography: started with and part of geodesy, this is a combination of procedures for direct land survey. Topography is a combination of methods and instruments to comprehensively measure and represent details of the Earth's surface:

- Planimetry: to determine the relative positions of the representation of points on the Earth's surface with respect to the same reference surface
- Altimetry: to determine the height of the points on the Earth's surface with respect to the geoid surface

- Tachymetry: for the planimetric and altimetric survey of the Earth's surface zones

- Land surveying: to measure areas, move and rectify borders, and level zones of the Earth's physical surface

- Cartography: to supply a possible description of the shape and dimension of the Earth and its natural and artificial details, by means of graphical or numerical representation of more or less wide areas, following fixed rules

- Photogrammetry: to determine the position and shapes of the objects by measuring them on photographic images

- Remote sensing: to remotely acquire territorial and environmental data and to combine methods and techniques for subsequent processing and interpretation (this definition also fits digital photogrammetry)

- Global positioning system (GPS): to provide the threedimensional position of fixed or moving objects, in space and time, all over the Earth's surface, under any meteorological conditions and in real time

- Laser scanning system: to locate objects and measure their distance by means of the incident radiation in the optical frequencies $(0.3-15 \mu \mathrm{m})$ of the electromagnetic spectrum

- Geographical information system (GIS): to make use of a powerful combination of instruments capable of receiving, recording, recalling, transforming, representing, and processing georeferenced spatial data

- Decision support system (DSS): to implement complex geographical information systems meant to create possible scenarios by modeling the ground truth and to offer a set of solutions to the decision maker

- Expert system: to consider instruments capable of imitating the experts' cognitive processes and their ability to manage the complexity of reality by means of interdependent processes of abstraction, generalization, and approximation

- WebGIS: to distribute geographic data remotely stored on dedicated machines for databases, according to complex network architectures

- Ontology: to specify a conceptuality, i.e., the description of concepts and relationships existing for an element or among various elements of a group, entity, or class; conceptualization is an abstract simplified vision of the world to be represented for a given application

\section{Computer science}

Computer science culture is now widespread, not only as regards technical instruments but also as regards methods, 
models, and systems that contribute to improving activities and research. Informatics, as a discipline, comprises both the computer technology, i.e., hardware or the physical components, and software, that is to say the way in which information is structured and elaborated.

The important role of information is derived from man's necessity to manage more and more numerous and complex data in every field. The scientific aspect of informatics is emphasized by the systematic, rigorous elaboration of information and its increasingly sophisticated automation.

The human brain constantly elaborates data through both simple and complex cognitive processes, often without any apparent effort. Every action is the result of a more or less complex elaboration by inputting continuously updated data, which are elaborated or simply stored for subsequent processing and then released as output data or information.

A close relationship exists between the possibility of data representation and data processing; human thinking is a form of calculation, and our mind outlines the truth through symbols and ideas. When a problem exceeds a certain degree of complexity and speed is of the essence, then human elaboration processes become problematic and, in some cases, cannot even be implemented.

The Association for Computing Machinery, an organization for informatics researchers and professionals, has issued the following definition to underline the planning and developing stages in any informatics activity:

...systematic study of the algorithms which describe

and transform information: their theory, analysis,

plan, efficiency, realization and application...

Algorithms are precise sequences of comprehensible operations, performable by an automatic instrument. Systems for automatic data elaboration are the result of a long process of evolution of elementary instruments for mechanical storage, invented by humans, in order to simplify the performance of calculations, from abacuses to the more recent mechanical calculators.

Current general-purpose devices may store sets of data, operations, and instructions, and they may perform such operations in succession, independently by working on data. Programs define the sequence of operations to be performed by expressing them in a language interpretable by the calculator.

\section{Data and information}

The two terms data and information are often used synonymously, while their individual meaning is in reality deeply different. The substantial distinction between them is comparable to the difference between an apparently disorganized set of letters and a word assembled with the same letters. Data are the basis of information and in general represent the measure of the external world. Only an expert system (whether human or not) is able to convert data into information, by reading it according to established rules. The acquisition of information goes via a cognitive process based on data.

In regard to geomatics, the following are a few possible examples:

- A raw satellite image represents the data and any finalized elaboration of it generates information (thematic images, topographical maps).

- GPS data are a measure of time; the derived information is a position in space.

- Records of an electronic archive or database are data; the answer to a query using data in a database generates information.

\section{Geodesy and cartography}

Representing the Earth in a synthetic and exhaustive way and as accurately as possible has been a great challenge for researchers since ancient times. In various ways and in line with the knowledge of the time, historical documentation presents geographic and territorial information using different media and picturesque techniques.

The maps are numerical-graphical products where the measure and understanding of a territory are synthetically reported. Nowadays, they are generally assembled with restitution procedures of aerial photogrammetry, providing a representation of the territory at a defined reduction scale, including planimetric details and shapes, and altimetric attributes.

Synthetically speaking, cartography can be defined as the representation of the Earth's surface based on specific rules. The International Association of Cartography (the world-leading institution for cartography, defined as a discipline dealing with the conception, production, distribution, and study of maps) has supplied the following definition:

A map is a symbolised image of geographic reality, representing selected features or characteristics, resulting from the creative effort of its author's choices, and it is designed for use when spatial relationships are of primary relevance (Sept. 1995).

Cartography takes advantage of traditional domains such as physics, geometry, design, geography, engineering, with the addition, during the last century, of methodological statistics and electronic numerical calculation for the rigorous elaboration of the data. 
Cartography also has a direct relationship with other disciplines for measuring and representing the physical surface of our planet "from its extensive complexity to its smaller detail": geodesy and topography. The relationships among these disciplines are shown in Fig. 1.

Geodesy is the science which defines the shape and dimensions of the Earth through its two branches:
- Gravimetry, for the determination of the Earth's gravity and its anomalies; the Earth's gravity determines the shape of the Earth, and the geoid is the equipotential surface of the gravitational field

- Positioning astronomy, for determining the position of the points on the globe through the observation of stars and artificial satellites, referring to the laws of celestial mechanics

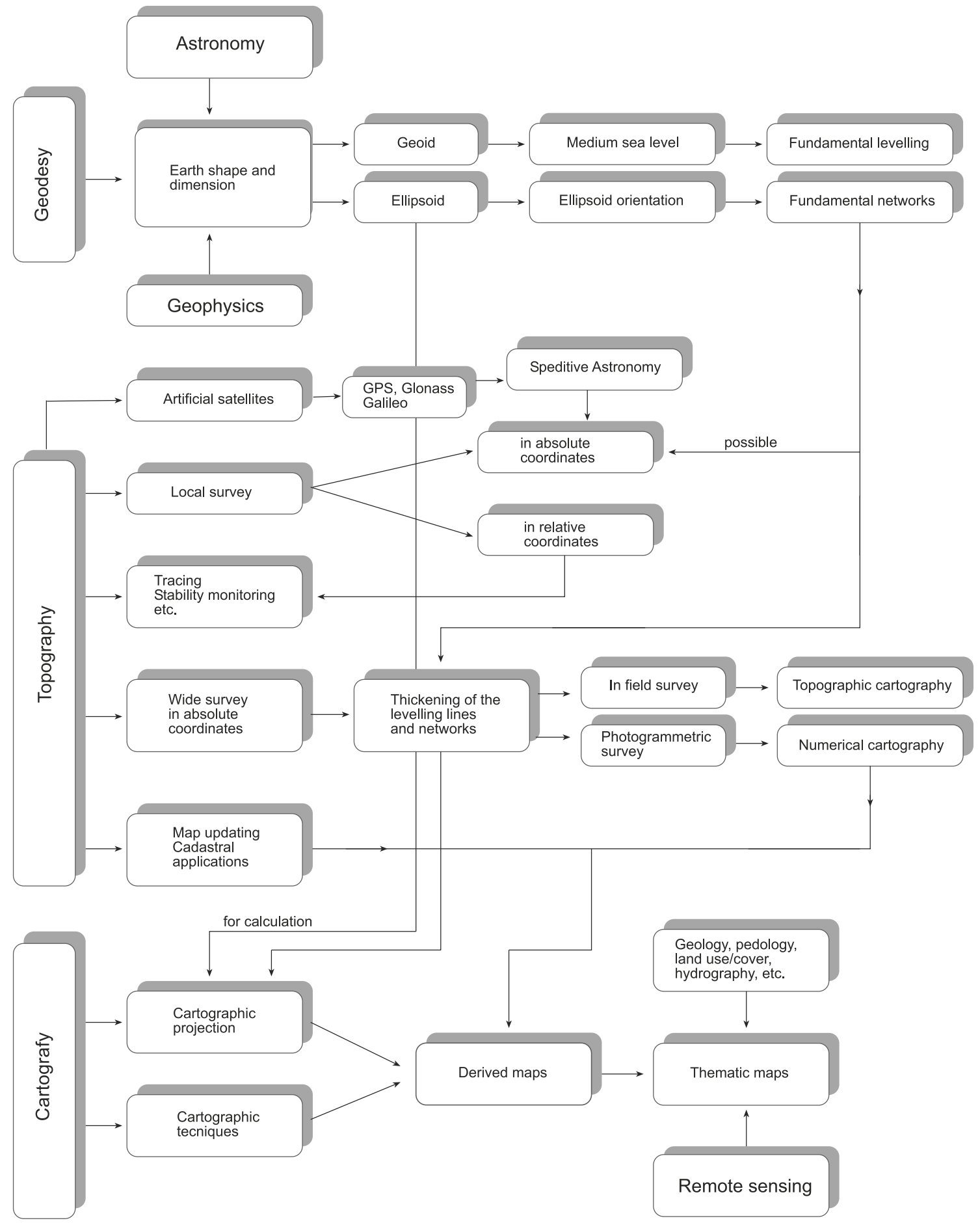

Fig. 1 Relationship among the most relevant elements of geomatics 
Topography (topo: place, graphia: writing) deals with the graphical and metric description of sites on the Earth's physical surface features. This discipline was defined as practical geometry until the eighteenth century to indicate its operating characteristics of survey and subsequent representation of the territory.

Photogrammetry, introduced in the nineteenth century, and remote sensing, introduced since the early 1970s, complete the picture.

Attention should be drawn to the use of the terms representation and projection of the planet Earth, which is a complex system that cannot be replaced with a geometric figure. It would be more correct to say representation on a plane of the reference surface rather than projection of the Earth. The representation of curved surfaces on a plane involves stretching, shrinking, and tearing, resulting in interruptions.

As a matter of fact, by applying the simple laws of projective geometry, an area of the Earth's surface is considered projected from a particular point of view using the three basic projection surfaces, plane, cylinder, or cone, in the so-called geometric projections.

\section{Photogrammetry (analogical, analytical, digital)}

Photogrammetry is defined as the process of deriving metric information about an object through measurements of the object made on photographs, leaving to photointerpretation (by human visual analysis) the task to obtain qualitative information (human experience remains a determinant factor).

With the introduction of technologies for image data acquisition from space in a wider region of the electromagnetic spectrum, the meaning of photogrammetry and photointerpretation has been extended to comprise remote sensing, moving from classical photointerpretation to the use of digital image processing in addition to human interpretation and applying computer analysis techniques to imagery besides photography.

A widely accepted definition of photogrammetry and remote sensing is the following:

Art, science and technology to obtain valid information about physical objects and the environment, through the processes of collection, measure and interpretation of images (photographic or digital) and analog or digital representation of the models of electromagnetic energy derived from survey systems (photographic cameras or scanning systems), without contact with the objects.

Traditional and digital photogrammetries are both based on the same fundamental principles. The tendency to use photogrammetry with high-resolution satellite data invites us to reconsider the traditional approach of the central perspective, while moving its formalisms towards more complex projective geometries that are more connected with the acquisition methods of satellite imagery and new aero-photogrammetric digital cameras. The rigorous reconstruction of the geometric correspondence between image and object at the moment of acquisition remains the primary scope of photogrammetry.

\section{Remote sensing}

Remote sensing includes techniques to derive information from a site at a known distance from the sensor. In passive remote sensing, the source of information is scattered and/ or absorbed solar and emitted thermal radiation, which allows us to study and characterize objects through their spectrally variable response. Every element on the Earth reflects, absorbs, and transmits part of an incident radiation to different percentages according to its structural, chemi$\mathrm{cal}$, and chromatic qualities. Electromagnetic radiance is the information reaching a sensor from the objects located on the Earth.

If in passive remote sensing the Sun is the most familiar source of energy, in active remote sensing, the sensor itself is at the same time emitter and receiver of electromagnetic energy. The principle used by the latter is the Radio Detection And Ranging effect and the palindrome well portrays the idea of emission and successive recording of the returning signal (backscattering) for determining the distance by means of electromagnetic waves with wavelength between $1 \mathrm{~mm}$ and $1 \mathrm{~m}$ (microwaves).

In common parlance, remote sensing, Télédétection (French), Fernerkundung (German), Perception Remota (Spanish), Sensoriamento Remoto (Portuguese), and Telerilevamento (Italian), indicates both the acquisition from a distance of qualitative and quantitative information concerning any site and the environment and the combination of methods and techniques for subsequent elaboration and interpretation. If the acquisition occurs from a short distance, the term proximal sensing is used.

The combination of measurable parameters from satellites and airplanes is widespread and applications of remote sensing in environmental sciences are scattered throughout the studies of biology, geochemistry, geology, mineral exploration, geomorphology, lithology, hydrology, oceanography, geobotany, classification of agricultural and forestry resources, environmental pollution, urban planning, risk management, permanent land monitoring, just to mention a few.

Remote sensing does not collect direct information on the environment so the electromagnetic information must 
be converted into estimates of chemical, biological, and physical variables through the creation of appropriate multidisciplinary models. The success or failure of remote sensing in estimating environmental alterations thus depends on models and algorithms developed and used to extract environmental parameters from the continuum of collected optical-spectral data from the sensors and on comparison with surface reference data assessment.

The collection and distribution of information relate to the development of sensor specifications, data transmission, and data processing. Sensors supply remote measures, basically observing the spectral behavior of the objects in the visible, infrared, and microwave electromagnetic intervals; such measures are used to indirectly adduce the structure of territorial elements or to survey some physical characteristics, like the temperature or spatial distribution of an element. In this regard, far remote sensing permits a qualitative and descriptive analysis of the images and, under defined conditions, a quantitative, automatic analysis.

Despite the many limitations of remote sensing, a considerable increase has been witnessed both in the number of missions and in the achievable geometric, temporal, radiometric, and spectral resolutions, which have enhanced its role in territorial planning, in managing land resources, and in monitoring environmental dynamic processes.

Satellite data concerning the same area are collected in a period that may vary from a few hours to some weeks. This provides the possibility to deliver an updated thematic cartography, where the only limit is represented by the geometric resolution of sensors with respect to the geometric precision of a map.

\section{Global satellite positioning systems}

The positioning systems applied to points on the Earth's surface have found practical application in topography and cartography, after their initial specific utilization in the field of ship navigation.

They allow the three-dimensional positioning of static or moving objects in space and time, in every place on the globe, under all meteorological conditions, and continuously.

They are based on the reception of radiofrequency signals emitted from artificial telecommunication satellites. The ground station must be equipped with an antenna and a receiver: their degree of complexity and cost depend on the measurement level of accuracy needed to determine geocentric coordinates (WGS84) of any point on the Earth's surface.

If we know the geocentric position of the satellites, whose orbits are referred to the geocentric reference system
WGS84, the geocentric coordinates can be directly converted by the receiver into other reference systems, supplying a three-dimensional positioning (3D).

Defining the position of a point is possible by calculating the distance between satellites and receiver, indirectly determined through measures of time or phase by exploiting the different characteristics of the signal emitted by the satellites and received by the receiver. The nature of such signals is defined from the positioning system of reference.

Two functioning constellations of positioning satellites, planned and launched in orbit during the 1970s and 1980s by the two former political blocs (USA and USSR), currently guarantee position measurements:

- American system NAVSTAR GPS, NAVigation Satellite Timing And Ranging Global Positioning System

- GLONASS, Global' naya Navigatsionnaya Sputnikovaya Sistema, currently managed by Russia

Europe is also working on an alternative constellation for global positioning with the aim of reducing dependence on American and Russian systems and to produce a more modern, reliable, and multifunctional system with wide strategic and economic benefits.

On July 19, 1999, the Council of Europe adopted a resolution to get the Galileo system underway in cooperation with the European Space Agency. This system is planned to have complete complementarity with the existing GPS and GLONASS systems.

\section{Laser scanning}

Among other survey disciplines, the laser scanning technique is particularly significant, as it is characterized by the ability to produce complete information and achieve high precision and by a considerable level of automation and productivity.

\section{Starting from a laser source, fixed or in motion, ground based or aerial, through the polar detection of a very large number of points surrounding the laser source and the radiometric measure of each of them, it is possible to recreate, nearly continuously, the three-dimensional image of the object or the surface of interest.}

The laser scanning techniques therefore represent a meaningful evolution of some aspects of photogrammetry, directly supplying a three-dimensional surface model, traditionally obtained from the stereoscopic elaboration of bidimensional images, thereby reducing the involvement of expert interpreters and approaching the total automation of the process.

Technology and computer science aspects characterize this technique, which offers multiple applications in ground 
and aerial surveys. Its weakness is represented by the complex and at present ill-defined filtering operations necessary to reduce and to select the enormous amount of data collected by the laser system and necessary to recreate the digital surface models.

\section{Geographical information systems}

As software for the management and manipulation of geographic data, the GIS appeared in the computer market in the mid-1960s. Progress in the field of GIS software has been rather slow in comparison with the information systems used for commercial or financial data. The delayed introduction of the GIS in the market can be explained by the fact that the data and information to be stored in such systems are more complex and difficult to process compared to other types of non-geographical information systems. Geospatial information, or geoinformation, in fact concerns phenomena referenced in planimetry and in altimetry, which are strongly interrelated. The widespread rapid diffusion of GIS starting in the 1990s has enormously increased retrieval, elaboration, and analysis capabilities of available data stored in the archives of public administrations, agencies, and research institutes that are fundamental for studying and planning the real world.

A GIS (in French: Sistème d'Information Géographique, SIG; in German: Geographisches Informationssystem; in Italian: Sistema Informativo Geografico) can be defined by focusing on the tool-base (Burrough), the organizational (Cowen), or the spatial database (Aronoff) aspects:

- Burrough (1986) first defined the GIS as a powerful set of tools for collecting, storing, retrieving at will, transforming and displaying spatial data from the real world.

- Cowen (1988) defined a GIS as a Decision Support System involving the integration of referenced spatial data in a problem solving environment.

- Aronoff (1989) considered the GIS as any manual or computer based set of procedures, used to store and manipulate geographically referenced data.

- Burrough (1997), again, defined the GIS as a powerful set of tools for collecting, storing, retrieving at will, transforming and displaying spatial data from the real world for a particular set of purposes.

The tool-base definition considers that geographic (or spatial) data represent phenomena from the real world in terms of:

- Position with respect to a known coordinate system

- Attributes when unrelated to position (name, age, height, etc.)
- Spatial interrelations with each other that describe how they are linked together (topology describing space and spatial properties; Burrough 1997)

\section{Decision support systems and expert systems}

Cowen's statement leads to a powerful evolution of the GIS, developing in DSS.

A synthesis of Burrough's and Cowen's statements can successfully summarize the evolution of the geographical information systems:

A Decision Support System (DSS) is a powerful set of tools for collecting, storing, retrieving at will, processing, transforming and displaying georeferenced spatial data in adequate scenarios of the real world so as to supply the decision makers with objective elements of evaluation of environmental problems.

By using DSS, one must be in a position to prevent and foresee territorial and environmental phenomena and to explore several scenarios to obtain an overview of their possible consequences. For example, the ability to foresee when a volcanic eruption or a flood event may happen, its intensity, and the extension of the area involved, may be useful in the definition of a plan for evacuating the population.

\section{Spatial information}

With the constitution of the GSDI Association, formally founded in July 2003, the term spatial data information was officially introduced as information related to the terrestrial globe in three-dimensional space.

The purpose of the GSDI organization is to promote international cooperation and collaboration in support of local, national, and international spatial data infrastructure developments that will allow nations to better address social, economic, and environmental issues of pressing importance.

However, some terminological nuances still remain. The common definition geographic information is used to indicate everything concerning the three-dimensional positioning and georeferencing of objects on our planet.

In some cases, the terms territorial information and geographic information are used as synonyms, although their different cultural backgrounds imply a distinction.

Geographic and territorial cultures are substantially different because of their humanistic or scientific approach, a distinction that still exists in some countries as between human geography and measurement of the territory. 
This difference is also reflected in expressions such as territorial information system and GIS, although the term GIS is accepted worldwide.

In this volume, reference will often be made to GeoSpatial Information as a good definition of the space we live in, increasingly measured, described, and represented in its three dimensions.

\section{Geography}

Geography possesses an intrinsic specificity and an epistemological autonomy such as to offer valid tools for an understanding of the real world.

The study of geography extends to a vast range of natural anthropical phenomena. Geography, in fact, based on the findings of physical and human sciences, performs an interpretative synthesis in order to analyze relationships, causes, effects, and evolutionary tendencies.

Geography is a qualifying and constructive means to achieve correct environmental and territorial policies (Fig. 2).

Anthropic geography studies the condition of humans on the territory and the relationships between organized human societies and natural environments, in the planetary geosystem and in each ecosystem at different scales. Perceptions, interactions, hierarchies, and different developments correspond to each of these; the location of whatever phenomenon cannot therefore be confined to an absolute analysis; rather, it must be seen in relation to increasing radius situations.

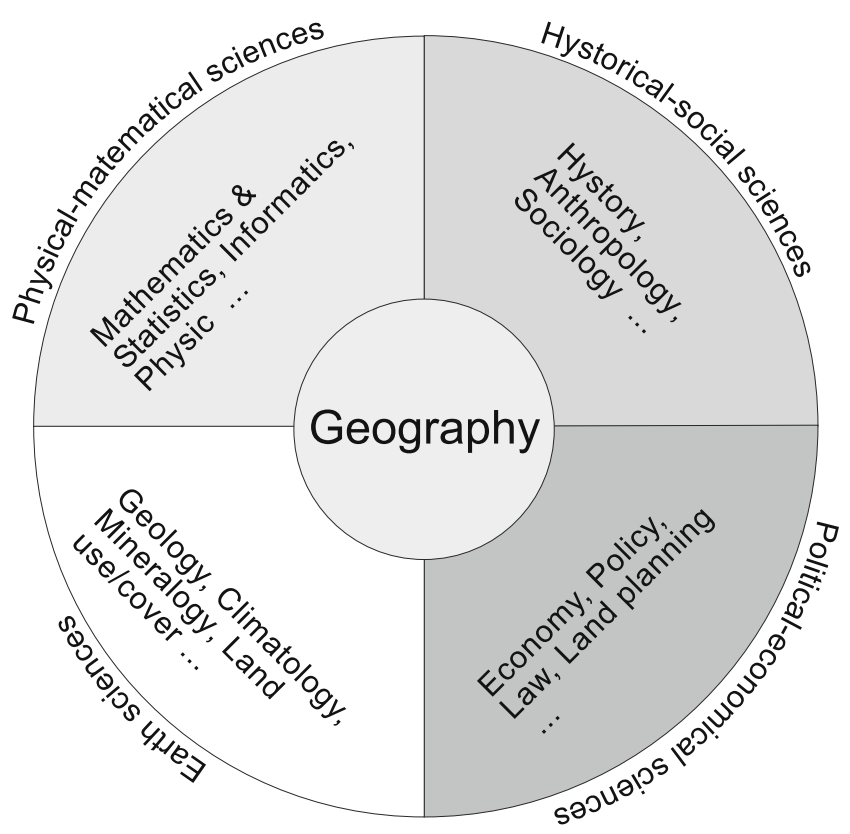

Fig. 2 The wheel of geography
Such a location must not be interpreted as the position of a place only; rather, it must encompass the concepts of distribution, association, and spatial specialization. In this way, distance can be not only metrical but also temporal, economical, and social.

\section{Ontology}

A brief section is devoted to ontology in this volume and in this chapter because of the growing interest it is attracting especially within the spatial information domain and because of its role in contributing to ordering terminologies and definitions often inadequately used as a consequence of the rapid development of geomatics.

The term ontology is often controversial in discussions of artificial intelligence. It dates back to the early stages of philosophy, with particular reference to the view of science as a historical fact and as an expression of human rationality. Ontology is very often confused with epistemology, and it is therefore worth explaining the philosophical meaning of the two terms:

- Epistemology: the study of nature and of the value of scientific knowledge, sustained by critical examination

- Ontology: also known as metaphysics, i.e., a philosophical doctrine related to the universal characters of the Supreme Being (God), corresponding to the older Aristotle's first philosophy. Ontology is the specification of conceptuality and the description of concepts and the relationships existing for an element or between elements of a group, entity, or class

The common denominator, of those who ventured into these thought traditions, is the account of theories of knowledge dealing with the history of scientific knowledge and with the development and consequent impact on society and the planet.

What is the role of ontology in geomatics? Ontology in geomatics implies the definition of different ontologies involved with sharing and reusing knowledge, so as to help define ontological assumptions.

An ontological assumption is the agreement to use a dictionary (asking questions and making statements) in a consistent but incomplete way, in comparison with the theory specified by ontology. Some elements reflect preset ontological principles. Sharing knowledge with and among these elements is possible thanks to the implemented ontologies.

The formal representation of knowledge is based on conceptualization: on the one hand, there are objects, concepts, and other entities assumed to exist in a certain domain of interest, while on the other hand we have the relationships established among them. The conceptualiza- 
tion is an abstract, simplified vision of the world that we wish to represent for a certain purpose. Every basic element of knowledge, every system based on knowledge, and every expert system is driven by an explicit or implicit conceptualization.

In artificial intelligence, what exists can also be represented. When knowledge within a certain domain is represented in a descriptive formalism, the group of objects to be represented is defined as universe of discourse. A dictionary is used to describe the relationships among objects belonging to the same group, which is also used to represent knowledge itself by means of a program based on knowledge.

In the context of artificial intelligence, it is possible to describe the ontology of a program by defining a group of representative terms for that application. In such an ontology, definitions associate the names of entities in the universe of discourse (for example, classes, relationships, functions, other objects, etc.) with a descriptive text showing the exact meaning of the terms, assuming formal axioms or limiting possible interpretations and use of such terms.

From a formal point of view, ontology is the definition of a logical theory.

The simplest example of ontology is the hierarchical taxonomic definition of classes in a legend (i.e., CORINE land cover).

\section{The geomatics expert}

The increasing interest in the field of spatial information, the complementarity, the integration, and the synergy among the disciplines and techniques characterizing it have recently led to the characterization of a new professional profile: the Geomatics Expert.

A similar professional profile already existed in North America at the beginning of the 1980s, whence it and progressively circulated in Europe and worldwide. It is the evolution of the Chartered Surveyor's profile in the English-speaking countries, of the Géométre Expert in the French-speaking countries, of the Vermessungsingenieur in the German-speaking countries, and of the Geodetische Ingenieur in the Netherlands.

A careful reading of the historical itinerary and different evolution processes identifies mathematics, physics, and particularly astronomy as the common origin of the survey disciplines, later to be elevated to sciences of land surveying starting in the seventeenth century.

The first differentiation takes place between the end of the eighteenth and the beginning of the nineteenth century in the "France of the Revolution and of the Empire," when the survey disciplines were included in the emerging field of civil engineering.
Only later did other differentiations occur at the European level.

The development of survey disciplines has progressed rapidly over recent decades: from spatial geodesy to precision topography, from photogrammetry to remote sensing, and from numerical cartography to the processing of observations and to the GIS, DSS, and expert systems.

Cartographic institutions have taken upon themselves official responsibility, increasingly extended, for the problem of quality certification (in survey and in projection).

The professional prospects for the expert in surveying and monitoring are therefore growing continuously and progressively. The professional profile of the geomatics expert is rapidly emerging, and this may correspond to a job opportunity if adequately supported by high-quality education and training.

Open Access This article is distributed under the terms of the Creative Commons Attribution Noncommercial License which permits any noncommercial use, distribution, and reproduction in any medium, provided the original author(s) and source are credited.

\section{References}

Annoni A (2003) INSPIRE: infrastructure of spatial information in Europe. In: 9th EC GIS\&GI A Coruna, Espana, 25-27 June 2003

Annoni A (2007) The contribution of INSPIRE to sustainable development. In: Gomarasca MA (ed) Proceedings of the 27th EARSeL Symposium GeoInformation in Europe. Millpress, Netherlands, pp 33-20. ISBN 9789059660618

Aronoff S (1989) Geographic information systems: a management perspective. WDL, Ottawa

Burrough PA (1986) Principles of geographical information systems for land resources assessment. Clarendon, Oxford

Burrough PA (2000) Principles of geographical information systems, spatial information systems and geostatistics. Claredon, Oxford, p $306 \mathrm{pp}$

Commission of the European Communities (2007) INSPIRE Directive, 2007/2/EC of the European Parliament and of the Council on March 14 establishing an infrastructure for spatial information in the European Community. Official Journal of the European Union 50:L108, ISSN 1725-2555

Cowen DJ (1988) GIS versus CAD versus DBMS: what are the differences? Photogramm Eng Remote Sens 54:1551-1554

Gomarasca MA (2000) Introduzione a Telerilevamento e GIS per la Gestione delle Risorse agricole e Ambientali, 2nd edn. AIT, Firenze, $250 \mathrm{pp}, 32$ Tavole a colori

Gomarasca MA (2004) Elementi di Geomatica. Associazione Italiana di Telerilevamento, Milan, $618 \mathrm{pp}$

Gomarasca MA (2007) International review of geo-spatial information and the 7th European Framework Programme. In: Gomarasca MA (ed) Proceedings of the 27th EARSeL Symposium, GeoInformation in Europe. Millpress, Netherlands, pp 3-11. ISBN 9789059660618

Gomarasca MA (ed) (2008) GeoInformation in Europe Proceedings of the 27th EARSeL Symposium. Millpress, Netherlands, 681 pp. ISBN 9789059660618

Gruber TR (1993a) A translation approach to portable ontologies. Knowl Acquis 5(2):199-220 
Gruber TR (1993) Toward principles for the design of ontologies used for knowledge sharing. Presented at the Padua workshop on formal ontology, March. Nicola Guarino Edited collection

Hofmann-Wellenhof B, Lichtenegger H, Wasle H (2008) GNSS - global navigation satellite systems: GPS, GLONASS, Galileo, and more. Springer, Heidelberg, 516 pp. ISBN 978-3-211-73012-6

ICA (1995) 17th International Cartographic Conference, 10th General Assembly of the International Cartographic Association, Barcelona, Spain, 3 September. Springer, Netherlands, ISSN: 0343-2521

Kraus K (1993) Fundamentals and standard processes. Photogrammetry, vol 1. Dummler, Bonn, $397 \mathrm{pp}$

Michalsky RS (1994) Inferential theory of learning: developing foundations for multistrategy learning. In: Michalski R, Tecuci
G (eds) Machine learning - a multistrategy approach, vol 4 . Morgan Kaufmann, San Francisco

Mikhail EM, Bethel JS, McGlone JC (2001) Introduction to modern photogrammetry. Wiley, New York

Philipson WR (1997) Manual of photographic interpretation, 2nd edn. American Society for Photogrammetry and Remote Sensing, Bethesda, pp 3-20

Richards JA, Jia X (1998) Remote sensing digital image analysis, an introduction, 3rd edn. Springer, Heidelberg, revisited and enlarged edition

Swain PH, Davis SM (1978) Remote sensing: the quantitative approach. McGraw-Hill, Parigi

Wolf PR (1988) Elements of photogrammetry, Chap 3: principles of photography. McGraw-Hill, New York, pp 41-60 